\title{
Green One-pot Synthesis of Novel Polysubstituted Pyrazole Derivatives as Potential Antimicrobial Agents
}

\section{Hamid Beyzaei, ${ }^{1, *}$ Zahra Motraghi, ${ }^{1}$ Reza Aryan, ${ }^{1}$ Mohammad Mehdi Zahedi ${ }^{2}$ and Alireza Samzadeh-Kermani ${ }^{1}$}

${ }^{1}$ Department of Chemistry, Faculty of Science, University of Zabol, Zabol, Iran

${ }^{2}$ Department of Chemistry, University of Saskatchewan, 110 Science Place, Saskatoon, SK S7N 5C9, Canada

*Corresponding author: E-mail: hbeyzaei@yahoo.com and hbeyzaei@uoz.ac.ir

Tel: +985431232186 / Fax: +985431232180

Received: 05-06-2017

\begin{abstract}
Various biological properties of natural and synthetic pyrazole derivatives such as anti-inflammatory, antimicrobial, neuroprotective, anticonvulsant, antidepressant and anticancer activities encouraged us to propose a new, fast, green and eco-friendly procedure for the preparation of some novel 5-amino-3-(aryl substituted)-1-(2,4-dinitrophenyl)-1H-pyrazole-4-carbonitriles. They were efficiently synthesized via one-pot two-step process reaction of malononitrile, 2,4-dinitrophenylhydrazine and different benzaldehydes in deep eutectic solvent (DES) glycerol/potassium carbonate. The products yield and reaction times were considerably improved in the presence of applied DES. Antibacterial effects of all newly synthesized pyrazoles in comparison with several common antibiotics were evaluated against a variety of Gram-positive and Gram-negative pathogenic bacteria. In addition to, their inhibitory activities on three fungi were compared to some current antifungal agents. The moderate to good antimicrobial potentials particularly against fungi were observed in the major heterocyclic compounds according to the IZD, MIC, MBC and MFC results.
\end{abstract}

Keywords: Green synthesis; deep eutectic solvent; glycerol/potassium carbonate; polysubstituted pyrazoles; antibacterial and antifungal activities

\section{Introduction}

Pyrazoles are an important class of azoles containing two adjacent nitrogen atoms, which are found as major or minor scaffolds in various medicinal compounds and natural products. L- $\alpha$-Amino- $\beta$-(pyrazolyl- $N$ )-propanoic acid and withasomnine, which were isolated from Citrullus vulgaris (watermelon) juice and from the roots of Withania somnifera Dun (Solanaceae), in fact, are two of the few naturally occurring pyrazoles that have found potential use as anti-diabetic and depressant agents in medicinal chemistry ${ }^{1,2}$ Pyrazofurin and formycin are natural $C$-nucleoside antibiotics that are used to treat viral infections as well as inhibition of tumor cells growth. Stanozolol is a synthetic anabolic steroid that can be applied for treatment of anaemia and hereditary angioedema. In addition to, the pyrazole ring as a part of the chemical structure of drugs such as antipyrine, celecoxib and betazole, plays an essential role in the relief of ear pain and swelling, improvement of osteoarthritis signs, and treatment of bacterial and fungal infections (Figure 1).

Compounds containing pyrazole moiety exhibit a wide variety of biological and pharmacological activities including analgesic, neuroprotective, anticonvulsant, angiotensin converting enzyme (ACE) inhibitory, anti-angiogenesis, antioxidant and antiviral activities. ${ }^{3-9}$ Numerous studies have also focused on antibacterial and antifungal properties of pyrazole derivatives. ${ }^{10-12}$ In a research project, inhibitory activities of some heterocyclic Schiff bases derived from thiocarbohydrazide were assessed against various pathogenic bacterial and fungal strains via measurement of their inhibition zone diameters. One of the synthesized 1,2,4-triazines could block the growth of all selected microorganism. ${ }^{13}$

Various methods were proposed for the synthesis of pyrazole and their analogues. ${ }^{14-17}$ In this regard, a solution of the appropriate triethylamine in 1,4-dioxane efficiently catalysed synthesis of pyridine, thiophene and $4 \mathrm{H}$-pyrane 


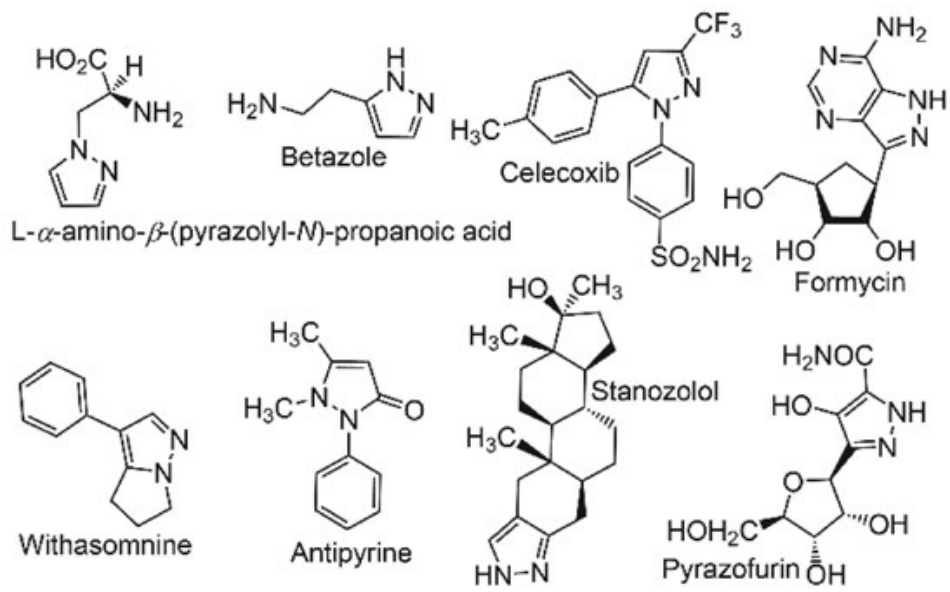

Figure 1. Natural products and drugs containing pyrazole moiety.

derivatives via one-pot or multicomponent protocols. ${ }^{18}$ Similar procedures were designed to prepare pyrazole derivatives. ${ }^{19-24}$ Most of these methods include simultaneous or multistep reaction of aldehyde, hydrazine and active methylene compounds under different conditions. ${ }^{25-29}$ Recently, deep eutectic solvents (DESs) were widely applied as eco-friendly media or efficient catalysts in organic synthesis especially for the preparation of pyrazoles. ${ }^{30-32}$ Glyc$\mathrm{erol} /$ potassium carbonate is a new class of DES having its physical properties, such as surface tension, viscosity, density and refractive index, carefully measured. ${ }^{33}$ In order to apply glycerol/ $/ \mathrm{K}_{2} \mathrm{CO}_{3}$ system in organic synthesis, some novel 5-amino-1-(2,4-dinitrophenyl)-1H-pyrazole-4-carbonitrile derivatives were prepared via the reaction of malononitrile, 2,4-dinitrophenylhydrazine and various benzaldehydes. The in vitro antimicrobial activities of synthesized derivatives were studied against a variety of pathogenic bacteria and fungi, as well as structure-activity relationships were expanded.

\section{Experimental}

\section{1. Chemicals}

All reagents, solvents, antibiotics and antifungal agents were purchased from commercial sources (Merck, Sigma and Aldrich), and used without further purification. The bacterial and fungal culture media were obtained from HiMedia. Melting points were determined with Kruss type KSP1N melting point meter and are uncorrected. Reaction progress was monitored by aluminium TLC plates pre-coated by silica gel with fluorescent indicator F254 using $\mathrm{CH}_{2} \mathrm{Cl}_{2} / \mathrm{CH}_{3} \mathrm{OH}(9: 1, \mathrm{v} / \mathrm{v})$ as the mobile phase, being visualized under UV radiation $(254 \mathrm{~nm})$. FT-IR spectra of the products were collected using Bruker Tensor-27 FT-IR spectrometer. ${ }^{1} \mathrm{H}$ and ${ }^{13} \mathrm{C}$ NMR spectra were recorded at 400 and $100 \mathrm{MHz}$, respectively, on a Bruker FT-NMR Ultra Shield-400 spectrometer. Elemental analyses were performed for $\mathrm{C}, \mathrm{H}$ and $\mathrm{N}$ on a Thermo Finnigan
Flash EA microanalyzer. DESs were prepared in various ratios of glycerol $/ \mathrm{K}_{2} \mathrm{CO}_{3}$ according to the procedures reported by Naser et al. ${ }^{33}$ as follows: the mixture of different molar ratios of potassium carbonate and glycerol were vigorously stirred at $80{ }^{\circ} \mathrm{C}$ for $2 \mathrm{~h}$ to gain homogenous transparent colorless liquids.

\section{1. 1. General Procedure for the Synthesis of Pyrazoles 4a-f}

A mixture of $\mathrm{K}_{2} \mathrm{CO}_{3}(0.140 \mathrm{~g}, 0.001 \mathrm{~mol})$ and glycerol $(0.360 \mathrm{~g}, 0.004 \mathrm{~mol})$ was stirred at $80{ }^{\circ} \mathrm{C}$ for $2 \mathrm{~h}$ to form a homogenous colorless liquid as DES1. Under the same conditions, the distilled water $(0.25 \mathrm{~mL})$, malononitrile (1) $(0.660 \mathrm{~g}, 0.001 \mathrm{~mol})$ and benzaldehydes $2 \mathrm{a}-\mathrm{f}(2 \mathrm{a}: 0.163 \mathrm{~g}$, 2b: 0.136 g, 2c: 0.151 g, 2d: 0.152 g, 2e: 0.175 g, 2f: 0.175 g; $0.001 \mathrm{~mol}$ ) were respectively added to it. The intermediate benzylidene malononitriles $\mathbf{6 a}-\mathbf{f}$ were produced in $2 \mathrm{~min}$. 2,4-Dinitrophenylhydrazine (3) $(0.198 \mathrm{~g}, 0.001 \mathrm{~mol})$ was added to the mixture. The reaction continued for another 18-28 min. The reaction mixture was cooled to room temperature, and neutralized with glacial acetic acid $(0.120 \mathrm{~g}$, $0.002 \mathrm{~mol}$ ). After adding $1 \mathrm{~mL}$ of ethanol, the mixture was poured into ice-cold saturated aqueous $\mathrm{NaCl}(5 \mathrm{~mL})$. The resulting precipitates were collected by filtration, washed respectively with distilled water $(5 \mathrm{~mL})$ and ethanol $(5$ $\mathrm{mL}$ ), and recrystallized from methanol to afford pure pyrazoles $\mathbf{4 a -} \mathbf{f}$ as colored crystals.

\section{1. 1. 1. $N$-(4-(5-Amino-4-cyano-1-(2,4-dinitrophenyl)- $1 \mathrm{H}$-pyrazol-3-yl)phenyl)acetamide (4a).}

Orange crystals; yield: $0.37 \mathrm{~g}$ (91\%); m.p. 274-275 ${ }^{\circ} \mathrm{C}$; IR (KBr) v 3444, $3281\left(\mathrm{NH}_{2}, \mathrm{NH}\right), 2231(\mathrm{C} \equiv \mathrm{N}), 1540$, $1326\left(\mathrm{NO}_{2}\right) \mathrm{cm}^{-1} ;{ }^{1} \mathrm{H}$ NMR $\left(400 \mathrm{MHz}, \mathrm{DMSO}-d_{6}\right) \delta 2.06(\mathrm{~s}$, $\left.3 \mathrm{H}, \mathrm{CH}_{3}\right), 7.75\left(\mathrm{~d}, J=8.7 \mathrm{~Hz}, 2 \mathrm{H}, \mathrm{H}-3^{\prime \prime}\right.$ and $\left.\mathrm{H}-5^{\prime \prime}\right), 7.87$ (d, $J=8.7 \mathrm{~Hz}, 2 \mathrm{H}, \mathrm{H}-2^{\prime \prime}$ and $\left.\mathrm{H}-6^{\prime \prime}\right), 7.98(\mathrm{~d}, J=8.5 \mathrm{~Hz}, 1 \mathrm{H}$, H-6'), 8.30 (d, $\left.J=8.5 \mathrm{~Hz}, 1 \mathrm{H}, \mathrm{H}-5^{\prime}\right), 8.55\left(\mathrm{~s}, 2 \mathrm{H}, \mathrm{NH}_{2}\right), 8.79$ (s, $\left.1 \mathrm{H}, \mathrm{H}-3^{\prime}\right), 10.48(\mathrm{~s}, 1 \mathrm{H}, \mathrm{NH}) ;{ }^{13} \mathrm{C}$ NMR $(100 \mathrm{MHz}$, DMSO- $\left.d_{6}\right) \delta 24.67\left(\mathrm{CH}_{3}\right), 78.00(\mathrm{C}-4), 114.21(\mathrm{C} \equiv \mathrm{N})$, 
119.20 (C-3" and C-5"), $123.43\left(\mathrm{C}-6^{\prime}\right), 126.15\left(\mathrm{C}-1^{\prime \prime}\right)$, $128.60\left(\mathrm{C}-5^{\prime}\right), 130.08\left(\mathrm{C}-3^{\prime}\right), 132.78\left(\mathrm{C}-2^{\prime \prime}\right.$ and C-6"'), $137.17\left(\mathrm{C}-1^{\prime}\right), 141.91\left(\mathrm{C}-2^{\prime}\right), 144.82\left(\mathrm{C}-4^{\prime}\right), 145.38\left(\mathrm{C}-4^{\prime \prime}\right)$, $149.71(\mathrm{C}-3), 160.67(\mathrm{C}-5), 169.77(\mathrm{C}=\mathrm{O})$. Anal. Calcd. for $\mathrm{C}_{18} \mathrm{H}_{13} \mathrm{~N}_{7} \mathrm{O}_{5}: \mathrm{C}, 53.07 ; \mathrm{H}, 3.22 ; \mathrm{N}, 24.07$. Found: $\mathrm{C}, 53.01$; $\mathrm{H}, 3.18 ; \mathrm{N}, 24.12$.

\section{1. 1. 2. 5-Amino-1-(2,4-dinitrophenyl)-3-(4- methoxyphenyl)- $1 \mathrm{H}$-pyrazole-4-carbonitrile (4b).}

Orange crystals; yield: $0.32 \mathrm{~g}(85 \%)$; m.p. $217-219^{\circ} \mathrm{C}$; IR $(\mathrm{KBr}) v$ 3456, $3326\left(\mathrm{NH}_{2}\right), 2224(\mathrm{C} \equiv \mathrm{N}), 1538,1319$ $\left(\mathrm{NO}_{2}\right) \mathrm{cm}^{-1} ;{ }^{1} \mathrm{H}$ NMR $\left(400 \mathrm{MHz}, \mathrm{DMSO}-d_{6}\right) \delta 3.88(\mathrm{~s}, 3 \mathrm{H}$, $\left.\mathrm{CH}_{3}\right), 7.03\left(\mathrm{~d}, J=8.7 \mathrm{~Hz}, 2 \mathrm{H}, \mathrm{H}-3^{\prime \prime}\right.$ and $\left.\mathrm{H}-5^{\prime \prime}\right), 7.71(\mathrm{~d}, J=$ $8.7 \mathrm{~Hz}, 2 \mathrm{H}, \mathrm{H}-2^{\prime \prime}$ and $\left.\mathrm{H}-6^{\prime \prime}\right), 8.01\left(\mathrm{~d}, J=8.4 \mathrm{~Hz}, 1 \mathrm{H}, \mathrm{H}-6^{\prime}\right)$, $8.38\left(\mathrm{~d}, J=8.4 \mathrm{~Hz}, 1 \mathrm{H}, \mathrm{H}-5^{\prime}\right), 8.62\left(\mathrm{~s}, 2 \mathrm{H}, \mathrm{NH}_{2}\right), 8.82(\mathrm{~s}$, $\left.1 \mathrm{H}, \mathrm{H}-3^{\prime}\right) ;{ }^{13} \mathrm{C}$ NMR $\left(100 \mathrm{MHz}\right.$, DMSO- $\left.d_{6}\right) \delta 56.39\left(\mathrm{CH}_{3}\right)$, $77.29(\mathrm{C}-4), 114.40(\mathrm{C} \equiv \mathrm{N}), 115.67\left(\mathrm{C}-3^{\prime \prime}\right.$ and $\left.\mathrm{C}-5^{\prime \prime}\right), 123.54$ (C-6'), $124.59\left(\mathrm{C}-1^{\prime \prime}\right), 127.97\left(\mathrm{C}-5^{\prime}\right), 130.04\left(\mathrm{C}-3^{\prime}\right), 133.84$ (C-2" and C-6"'), 137.13 (C-1'), $141.07\left(\mathrm{C}-2^{\prime}\right), 144.93$ (C-4'), 149.62 (C-3), 160.91 (C-5), 164.82 (C-4"). Anal. Calcd. for $\mathrm{C}_{17} \mathrm{H}_{12} \mathrm{~N}_{6} \mathrm{O}_{5}: \mathrm{C}, 53.69 ; \mathrm{H}, 3.18 ; \mathrm{N}, 22.10$. Found: C, 53.64; H, 3.17; N, 22.10 .

\section{1. 1. 3. 5-Amino-1-(2,4-dinitrophenyl)-3-(4- nitrophenyl)-1H-pyrazole-4-carbonitrile (4c).} Yellow crystals; yield: $0.35 \mathrm{~g}(89 \%)$; m.p. $295-296^{\circ} \mathrm{C}$; IR $(\mathrm{KBr}) v 3445,3325\left(\mathrm{NH}_{2}\right), 2228(\mathrm{C} \equiv \mathrm{N}), 1543,1318$ $\left(\mathrm{NO}_{2}\right) \mathrm{cm}^{-1} ;{ }^{1} \mathrm{H}$ NMR $\left(400 \mathrm{MHz}, \mathrm{DMSO}-d_{6}\right) \delta 8.06(\mathrm{~d}, J=$ $\left.9.3 \mathrm{~Hz}, 1 \mathrm{H}, \mathrm{H}-6^{\prime}\right), 8.15\left(\mathrm{~d}, J=7.9 \mathrm{~Hz}, 2 \mathrm{H}, \mathrm{H}-2^{\prime \prime}\right.$ and H-6"), $8.24\left(\mathrm{~d}, J=7.9 \mathrm{~Hz}, 2 \mathrm{H}, \mathrm{H}-3^{\prime \prime}\right.$ and $\left.\mathrm{H}-5^{\prime \prime}\right), 8.38$ (d, $J$ $\left.=9.3 \mathrm{~Hz}, 1 \mathrm{H}, \mathrm{H}-5^{\prime}\right), 8.48\left(\mathrm{~s}, 2 \mathrm{H}, \mathrm{NH}_{2}\right), 8.81\left(\mathrm{~s}, 1 \mathrm{H}, \mathrm{H}-3^{\prime}\right)$; ${ }^{13} \mathrm{C}$ NMR (100 MHz, DMSO- $\left.d_{6}\right) \delta 89.02(\mathrm{C}-4), 117.37$ $(\mathrm{C} \equiv \mathrm{N}), 121.69\left(\mathrm{C}-2^{\prime \prime}\right.$ and $\left.\mathrm{C}-6^{\prime \prime}\right), 123.34\left(\mathrm{C}-6^{\prime}\right), 125.10$ (C-3" and C-5"), 130.35 (C-5'), $131.62\left(\mathrm{C}-3^{\prime}\right), 136.32$ (C-1'), 138.05 (C-1"'), 142.82 (C-2'), $144.70\left(\mathrm{C}-4^{\prime}\right), 147.29$ (C-4"), 148.80 (C-3), 159.75 (C-5). Anal. Calcd. for $\mathrm{C}_{16} \mathrm{H}_{9} \mathrm{~N}_{7} \mathrm{O}_{6}: \mathrm{C}, 48.62 ; \mathrm{H}, 2.30 ; \mathrm{N}, 24.80$. Found: C, 48.68; $\mathrm{H}, 2.25 ; \mathrm{N}, 24.84$.

\section{1. 1. 4. 5-Amino-1-(2,4-dinitrophenyl)-3-(2-hydroxy- 3-methoxyphenyl)- $1 \mathrm{H}$-pyrazole-4-carbonitrile} (4d).

Brown crystals; yield: $0.33 \mathrm{~g}(84 \%)$; m.p. $198-199^{\circ} \mathrm{C}$; IR $(\mathrm{KBr}) v 3537(\mathrm{OH}), 3428,3287\left(\mathrm{NH}_{2}\right), 2206(\mathrm{C} \equiv \mathrm{N})$, 1517, $1331\left(\mathrm{NO}_{2}\right) \mathrm{cm}^{-1} ;{ }^{1} \mathrm{H}$ NMR $\left(400 \mathrm{MHz}\right.$, DMSO- $\left.d_{6}\right) \delta$ $3.77\left(\mathrm{~s}, 3 \mathrm{H}, \mathrm{CH}_{3}\right), 6.76\left(\mathrm{~m}, 1 \mathrm{H}, \mathrm{H}-4^{\prime \prime}\right), 6.93\left(\mathrm{~m}, 1 \mathrm{H}, \mathrm{H}-5^{\prime \prime}\right)$, $7.31\left(\mathrm{~m}, 1 \mathrm{H}, \mathrm{H}-6^{\prime \prime}\right), 7.91\left(\mathrm{~d}, J=8.1 \mathrm{~Hz}, 1 \mathrm{H}, \mathrm{H}-6^{\prime}\right), 8.24$ (d, $\left.J=8.1 \mathrm{~Hz}, 1 \mathrm{H}, \mathrm{H}-5^{\prime}\right), 8.87$ (s, $\left.1 \mathrm{H}, \mathrm{H}-3^{\prime}\right), 9.43\left(\mathrm{~s}, 2 \mathrm{H}, \mathrm{NH}_{2}\right.$ ), $11.63(\mathrm{~s}, 1 \mathrm{H}, \mathrm{OH}) ;{ }^{13} \mathrm{C}$ NMR $\left(100 \mathrm{MHz}, \mathrm{DMSO}-d_{6}\right) \delta 56.23$ $\left(\mathrm{CH}_{3}\right), 84.75(\mathrm{C}-4), 113.67(\mathrm{C} \equiv \mathrm{N}), 116.95\left(\mathrm{C}-4^{\prime \prime}\right), 118.12$ (C-1"), $119.58\left(\mathrm{C}-5^{\prime \prime}\right), 120.71$ (C-6"), 123.31 (C-6'), 129.44 (C-5'), 129.99 (C-3'), 137.05 (C-1'), 140.90 (C-2'), 142.15 (C-2"), 144.64 (C-4'), 146.43 (C-3), 148.43 (C-3"), 162.71 (C-5). Anal. Calcd. for $\mathrm{C}_{17} \mathrm{H}_{12} \mathrm{~N}_{6} \mathrm{O}_{6}$ : C, 51.52; $\mathrm{H}, 3.05 ; \mathrm{N}$, 21.21. Found: C, 51.45; H, 3.11; N, 21.18.
2. 1. 1. 5. 5-Amino-3-(2,4-dichlorophenyl)-1-(2,4dinitrophenyl)- $1 \mathrm{H}$-pyrazole-4-carbonitrile (4e).

Yellow crystals; yield: $0.36 \mathrm{~g}(86 \%)$; m.p. $184-186^{\circ} \mathrm{C}$; IR $(\mathrm{KBr}) v 3443,3287\left(\mathrm{NH}_{2}\right), 2227(\mathrm{C} \equiv \mathrm{N}), 1514,1330$ $\left(\mathrm{NO}_{2}\right) \mathrm{cm}^{-1} ;{ }^{1} \mathrm{H}$ NMR $\left(400 \mathrm{MHz}, \mathrm{DMSO}-d_{6}\right) \delta 7.46(\mathrm{~d}, J=$ $\left.5.8 \mathrm{~Hz}, 1 \mathrm{H}, \mathrm{H}-5^{\prime \prime}\right), 7.66$ (s, $\left.1 \mathrm{H}, \mathrm{H}-3^{\prime \prime}\right), 7.86$ (d, $J=5.8 \mathrm{~Hz}$, $\left.1 \mathrm{H}, \mathrm{H}-6^{\prime \prime}\right), 8.01$ (d, $\left.J=8.0 \mathrm{~Hz}, 1 \mathrm{H}, \mathrm{H}-6^{\prime}\right), 8.29$ (d, $J=8.0$ $\left.\mathrm{Hz}, 1 \mathrm{H}, \mathrm{H}-5^{\prime}\right), 8.77$ (s, $\left.1 \mathrm{H}, \mathrm{H}-3^{\prime}\right), 8.98\left(\mathrm{~s}, 2 \mathrm{H}, \mathrm{NH}_{2}\right) ;{ }^{13} \mathrm{C}$ NMR $\left(100 \mathrm{MHz}, \mathrm{DMSO}-d_{6}\right) \delta 87.52(\mathrm{C}-4), 113.76(\mathrm{C} \equiv \mathrm{N})$, $123.21\left(\mathrm{C}-6^{\prime}\right), 128.39$ (C-5'), $128.77\left(\mathrm{C}-5^{\prime \prime}\right), 129.04\left(\mathrm{C}-1^{\prime \prime}\right)$, $130.06\left(\mathrm{C}-3^{\prime}\right), 130.48\left(\mathrm{C}-6^{\prime \prime}\right), 131.40\left(\mathrm{C}-3^{\prime \prime}\right), 134.55\left(\mathrm{C}-2^{\prime \prime}\right)$, $135.81\left(\mathrm{C}-4^{\prime \prime}\right), 137.95\left(\mathrm{C}-1^{\prime}\right), 139.16\left(\mathrm{C}-2^{\prime}\right), 144.37\left(\mathrm{C}-4^{\prime}\right)$, 144.65 (C-3), 157.17 (C-5). Anal. Calcd. for $\mathrm{C}_{16} \mathrm{H}_{8} \mathrm{Cl}_{2} \mathrm{~N}_{6} \mathrm{O}_{4}$ : C, 45.85; H, 1.92; N, 20.05. Found: C, 45.90; H, 1.89; N, 19.98 .

\section{1. 1. 6. 5-Amino-3-(2,6-dichlorophenyl)-1-(2,4- dinitrophenyl)- $1 \mathrm{H}$-pyrazole-4-carbonitrile (4f).}

Orange crystals; yield: $0.37 \mathrm{~g}(88 \%)$; m.p. $256-257^{\circ} \mathrm{C}$; IR $(\mathrm{KBr}) v 3443,3287\left(\mathrm{NH}_{2}\right), 2227(\mathrm{C} \equiv \mathrm{N}), 1514,1330$ $\left(\mathrm{NO}_{2}\right) \mathrm{cm}^{-1} ;{ }^{1} \mathrm{H}$ NMR $\left(400 \mathrm{MHz}, \mathrm{DMSO}-d_{6}\right) \delta 7.44(\mathrm{~d}, J=$ $\left.6.1 \mathrm{~Hz}, 1 \mathrm{H}, \mathrm{H}-4^{\prime \prime}\right), 7.58$ (t, $\left.J=6.1 \mathrm{~Hz}, 2 \mathrm{H}, \mathrm{H}-3^{\prime \prime}\right), 7.96$ (d, $J$ $\left.=8.4 \mathrm{~Hz}, 1 \mathrm{H}, \mathrm{H}-6^{\prime}\right), 8.40\left(\mathrm{~d}, J=8.4 \mathrm{~Hz}, 1 \mathrm{H}, \mathrm{H}-5^{\prime}\right), 8.83(\mathrm{~s}$, $\left.1 \mathrm{H}, \mathrm{H}-3^{\prime}\right), 9.01\left(\mathrm{~s}, 2 \mathrm{H}, \mathrm{NH}_{2}\right) ;{ }^{13} \mathrm{C}$ NMR $(100 \mathrm{MHz}, \mathrm{DM}-$ SO- $\left.d_{6}\right) \delta 84.86(\mathrm{C}-4), 117.36(\mathrm{C} \equiv \mathrm{N}), 123.28\left(\mathrm{C}-6^{\prime}\right), 126.36$ $\left(\mathrm{C}-1^{\prime \prime}\right), 130.48\left(\mathrm{C}-5^{\prime}\right), 130.06\left(\mathrm{C}-3^{\prime \prime}\right.$ and $\left.\mathrm{C}-5^{\prime \prime}\right), 130.61$ $\left(\mathrm{C}-4^{\prime \prime}\right), 131.41\left(\mathrm{C}-3^{\prime}\right), 131.64\left(\mathrm{C}-2^{\prime \prime}\right.$ and $\left.\mathrm{C}-6^{\prime \prime}\right), 138.21$ (C-1'), 140.90 (C-2'), 144.35 (C-4'), 144.89 (C-3), 158.66 (C-5). Anal. Calcd. for $\mathrm{C}_{16} \mathrm{H}_{8} \mathrm{Cl}_{2} \mathrm{~N}_{6} \mathrm{O}_{4}: \mathrm{C}, 45.85 ; \mathrm{H}, 1.92 ; \mathrm{N}$, 20.05. Found: C, $45.81 ; \mathrm{H}, 1.87 ; \mathrm{N}, 20.10$.

\section{2. In vitro Antimicrobial Assay}

Gram-negative bacterial strains including Pseudomonas aeruginosa (PTCC 1310), Shigella flexneri (PTCC 1234), Shigella dysenteriae (PTCC 1188), Proteus mirabilis (PTCC 1776), Proteus vulgaris (PTCC 1079), Salmonella enterica subsp. enterica (PTCC 1709) and Salmonella typhi (PTCC 1609); Gram-positive bacterial strains including Streptococcus pyogenes (PTCC 1447), Streptococcus agalactiae (PTCC 1768), Streptococcus pneumoniae (PTCC 1240), Staphylococcus epidermidis (PTCC 1435) and Rhodococcus equi (PTCC 1633); and fungi including Aspergillus fumigatus (PTCC 5009), Candida albicans (PTCC 5027) and Fusarium oxysporum (PTCC 5115) were prepared from the Persian Type Culture Collection (PTCC), Tehran, Iran. Initial concentrations of $17.6 \mu \mathrm{g} / \mathrm{mL}$ of positive controls were prepared in double-distilled water. Accordingly, heterocyclic compounds were dissolved in $10 \%$ DMSO to produce final concentrations of $10240 \mu \mathrm{g} / \mathrm{mL}$. All the antibiogram assays were repeated at least three times. The results were reported as the mean of three independent experiments. Antibacterial and antifungal activities were determined using both broth microdilution and 
disk diffusion methods, according to Clinical and Laboratory Standards Institute (CLSI) guidelines M07-A9, M26-A, M02-A11, M44-A and M27-A2 with a slight modification. $^{34,35}$

\section{Results and Discussion}

\section{1. Chemistry}

In this project, 5-amino- $1 H$-pyrazole-4-carbonitriles were synthesized via an efficient, environmentally friendly, cost-effective and fast process. One-pot two-step reaction of malononitrile (1), mono or disubstituted benzaldehydes 2a-f and 2,4-dinitrophenylhydrazine (3) produced polyfunctional pyrazoles $\mathbf{4 a}-\mathbf{f}$ in high yields (Scheme 1). The best results were obtained with glycerol/ $/ \mathrm{K}_{2} \mathrm{CO}_{3}$ as the reaction media and catalyst.

The reaction conditions were optimized in terms of solvent, presence or absence of the catalyst and temperature. $1 \mathrm{mmol}$ each of malononitrile (1), 4-acetamidobenzaldehyde (2a) and 2,4-dinitrophenylhydrazine (3) were reacted under different conditions (Table 1). Glycer$\mathrm{ol}$ as a green, cheap, non-toxic, inflammable and readily available solvent was the component present in all reactions. No target products were obtained when the reaction mixture was stirred at room temperature. The solubility of reagents was improved as the viscosity of glycerol largely decreased with increasing temperature to $80^{\circ} \mathrm{C}$. All efforts to perform three-component reaction in media containing glycerol alone were unsuccessful (Entries $1,2)$. The formation of Schiff bases as major products in glycerol showed that the presence of $\mathrm{K}_{2} \mathrm{CO}_{3}$ catalyst is required for the synthesis of pyrazoles (Entries 3-6). There are two possible mechanisms to form the products, but it seems that only route $b$ will afford the final compounds $\mathbf{4 a}-\mathbf{f}$ (Scheme 2). Schiff-base condensation reaction was observed in route $a$ under some of the applied conditions. Colorless solutions of various molar ratios of potassium carbonate to glycerol (DES1, 1:4; DES2, 1:5; DES3, 1:6) were selected because their physical properties, including conductivity, surface tension, viscosity, refractive index, density and $\mathrm{pH}$ have been evaluated very well in the temperature range of $10-80{ }^{\circ} \mathrm{C} .{ }^{33}$ Three-component reaction in $0.5 \mathrm{~g}$ of each of these three DESs at room temperature have resulted in the Schiff bases and benzylidenemalononitriles as the major products (Entries 7, 9, 11), pyrazoles were obtained in $35-40 \%$ yields due to the increase of temperature to $80^{\circ} \mathrm{C}$ (Entries $8,10,12$ ). One-pot twostep process was screened according to route $b$. Two-step procedure was carried out in all DESs, the increase in molar ratios of glycerol reduced the product yield (Entries 13-18). This can be caused by the higher $\mathrm{pH}$ of DES1. The first stage reaction did not proceed completely at room temperature or at higher temperature even after $8 \mathrm{~h}$, due to the lack of appearance of the intermediary benzylidenemalononitriles in the reaction media. Therefore, the next stage reaction of unconsumed reagents especially aldehydes with hydrazine was inevitable under these conditions (Entries 13, 15, 17). Adding water to achieve the final DES1/ $\mathrm{H}_{2} \mathrm{O}$ ratios of $1: 2,1: 1$ and $3: 1$ (w:w) has improved reaction time and product yield, the condensation reaction of malononitrile with aldehyde

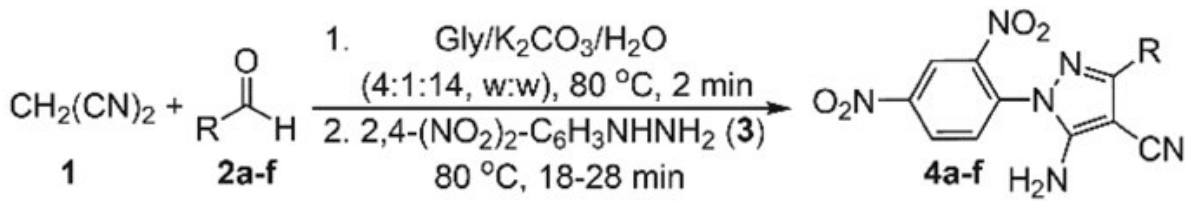

$\mathrm{R}=\mathbf{a}: 4-\mathrm{CH}_{3} \mathrm{CONH}-\mathrm{C}_{6} \mathrm{H}_{4}, \mathbf{b}: 4-\mathrm{CH}_{3} \mathrm{O}-\mathrm{C}_{6} \mathrm{H}_{4}, \mathbf{c}: 4-\mathrm{O}_{2} \mathrm{~N}-\mathrm{C}_{6} \mathrm{H}_{4}$,

d: $2-\mathrm{HO}-3-\mathrm{CH}_{3} \mathrm{O}-\mathrm{C}_{6} \mathrm{H}_{3}$, e: $2,4-\mathrm{Cl}_{2}-\mathrm{C}_{6} \mathrm{H}_{3}$, f: $2,6-\mathrm{Cl}_{2}-\mathrm{C}_{6} \mathrm{H}_{3}$

Scheme 1. Total synthesis of polysubstituted pyrazoles $\mathbf{4 a - f}$.

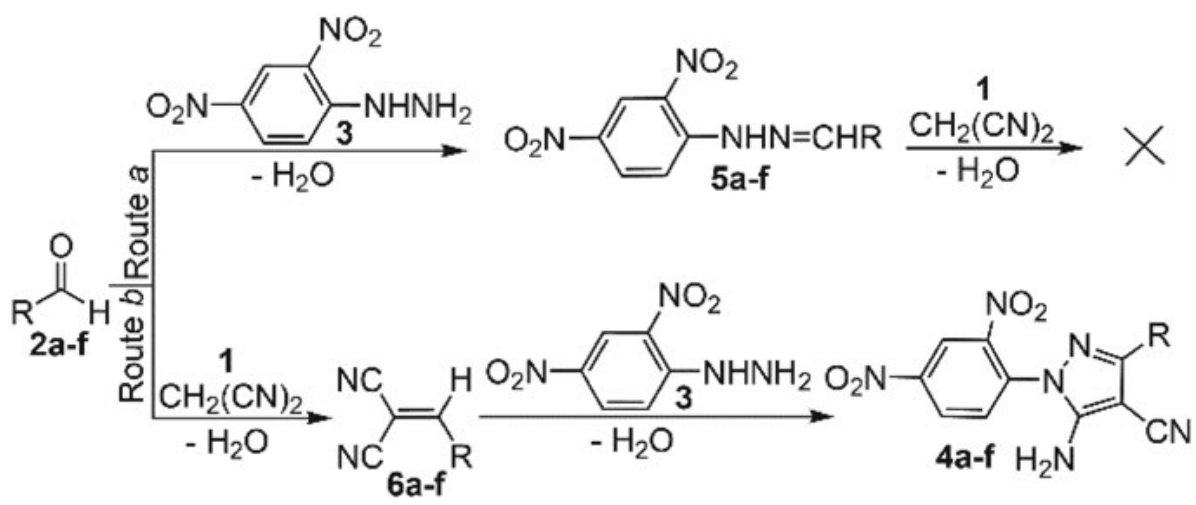

Scheme 2. Proposed mechanisms for the formation of pyrazole derivatives $4 \mathbf{a}-\mathbf{f}$. 
Table 1. Optimization of the model reaction conditions.

\begin{tabular}{|c|c|c|c|c|}
\hline Entry & Solvent & Condition & Time (min) & Yield (\%) \\
\hline 1 & Gly $^{\mathrm{a}}$ & Three-component, rt & 240 & - \\
\hline 2 & Gly & Three-component, $80^{\circ} \mathrm{C}$ & 120 & Schiff base \\
\hline 3 & Gly & One-pot two-step process (route $a$ ), rt & 240 & - \\
\hline 4 & Gly & One-pot two-step process (route $a$ ), $80^{\circ} \mathrm{C}$ & 180 & Schiff base \\
\hline 5 & Gly & One-pot two-step process (route $b$ ), rt & 240 & - \\
\hline 6 & Gly & One-pot two-step process (route $b$ ), $80^{\circ} \mathrm{C}$ & 180 & Schiff base \\
\hline 7 & DES1 & Three-component, rt & 210 & Benzylidene, Schiff base \\
\hline 8 & DES1 & Three-component, $80^{\circ} \mathrm{C}$ & 90 & 40 \\
\hline 9 & DES2 & Three-component, rt & 180 & Benzylidene, Schiff base \\
\hline 10 & DES2 & Three-component, $80^{\circ} \mathrm{C}$ & 90 & 37 \\
\hline 11 & DES3 & Three-component, rt & 150 & Benzylidene, Schiff base \\
\hline 12 & DES3 & Three-component, $80^{\circ} \mathrm{C}$ & 90 & 35 \\
\hline 13 & DES1 & One-pot two-step process (route $b$ ), rt & 180 & Benzylidene, Schiff base \\
\hline 14 & DES1 & One-pot two-step process (route $b$ ), $80^{\circ} \mathrm{C}$ & 120 & 53 \\
\hline 15 & DES2 & One-pot two-step process (route $b$ ), rt & 150 & Benzylidene, Schiff base \\
\hline 16 & DES2 & One-pot two-step process (route $b$ ), $80^{\circ} \mathrm{C}$ & 150 & 47 \\
\hline 17 & DES3 & One-pot two-step process (route $b$ ), rt & 120 & Benzylidene, Schiff base \\
\hline 18 & DES3 & One-pot two-step process (route $b$ ), $80^{\circ} \mathrm{C}$ & 180 & 42 \\
\hline 19 & $\mathrm{DES} 1 / \mathrm{H}_{2} \mathrm{O}, 1: 2^{\mathrm{b}}$ & One-pot two-step process (route $b$ ), $80^{\circ} \mathrm{C}$ & 50 & 60 \\
\hline 20 & $\mathrm{DES} 1 / \mathrm{H}_{2}^{2} \mathrm{O}, 1: 1^{\mathrm{b}}$ & One-pot two-step process (route $b$ ), $80^{\circ} \mathrm{C}$ & 35 & 75 \\
\hline 21 & $\mathrm{DES} 1 / \mathrm{H}_{2}^{2} \mathrm{O}, 2: 1^{\mathrm{b}}$ & One-pot two-step process (route $b$ ), $80^{\circ} \mathrm{C}$ & 20 & 91 \\
\hline 22 & $\mathrm{DES} 1 / \mathrm{H}_{2} \mathrm{O}, 3: 1^{\mathrm{b}}$ & One-pot two-step process (route $b$ ), $80^{\circ} \mathrm{C}$ & 40 & 68 \\
\hline
\end{tabular}

${ }^{\mathrm{a}}$ Gly as glycerol; ${ }^{\mathrm{b}}$ Ratios as w:w; The amount $0.5 \mathrm{~g}$ of solvents containing glycerol was used. Under the optimized conditions, mono and disubstituted benzaldehydes $\mathbf{2 b}-\mathbf{f}$ were also reacted with malononitrile (1) and 2,4-dinitrophenylhydrazine (3) to afford pyrazoles $\mathbf{4 b}-\mathbf{f}$. The results are presented in Table 2.

Table 2. Synthesis of polysubstituted pyrazoles $\mathbf{4 a}-\mathbf{f}$ under optimized conditions.

\begin{tabular}{lcccc}
\hline Entry & $\mathbf{R}$ & Product & $\begin{array}{c}\text { Time } \\
(\mathbf{m i n})\end{array}$ & $\begin{array}{c}\text { Yield }^{\mathbf{a}} \\
(\mathbf{\%})\end{array}$ \\
\hline 1 & $4-\mathrm{CH}_{3} \mathrm{CONH}-\mathrm{C}_{6} \mathrm{H}_{4}$ & $\mathbf{4 a}$ & 20 & 91 \\
2 & $4-\mathrm{CH}_{3} \mathrm{O}-\mathrm{C}_{6} \mathrm{H}_{4}$ & $\mathbf{4 b}$ & 25 & 85 \\
3 & $4-\mathrm{O}_{2} \mathrm{~N}_{4} \mathrm{C}_{6} \mathrm{H}_{4}$ & $\mathbf{4 c}$ & 20 & 89 \\
4 & $2-\mathrm{HO}_{4}-\mathrm{CH}_{3} \mathrm{O}_{-}-\mathrm{C}_{6} \mathrm{H}_{3}$ & $\mathbf{4 d}$ & 30 & 84 \\
5 & $2,4-\mathrm{Cl}_{2}-\mathrm{C}_{6} \mathrm{H}_{3}$ & $\mathbf{4 e}$ & 25 & 86 \\
6 & $2,6-\mathrm{Cl}_{2}-\mathrm{C}_{6} \mathrm{H}_{3}$ & $\mathbf{4 f}$ & 25 & 88 \\
\hline
\end{tabular}

${ }^{a}$ All yields refer to isolated products

was thus completed within 2 min (Entries 19, 20,22). The best results were obtained with $\mathrm{DES} 1 / \mathrm{H}_{2} \mathrm{O}$ ratio of $2: 1$ $(\mathrm{w}: \mathrm{w})$, and this was considered as the optimized conditions (Entry 21).

The molecular structures and purity of the newly synthesized compounds were identified by NMR $\left({ }^{1} \mathrm{H}\right.$ and ${ }^{13} \mathrm{C}$ ), FT-IR and elemental analysis (CHN). In FT-IR spectra, absorption bands attributed to symmetric and asymmetric stretching vibrations of amino groups appeared within $v=3428-3456$ and $3281-3326 \mathrm{~cm}^{-1}$, as well as stretching vibrations of nitro groups were recorded within $v=1514-1543$ and $1318-1331 \mathrm{~cm}^{-1}$. The presence of nitrile groups was deduced both from IR bonds and ${ }^{13} \mathrm{C}$ NMR signals appearing at $v=2206-2228 \mathrm{~cm}^{-1}$ and $\delta$
113.67-117.37 ppm. In addition to these, ${ }^{1} \mathrm{H}$ NMR spectra and microanalytical data are in agreement with the chemical structures.

\section{2. Antimicrobial Evaluation}

The in vitro inhibitory activities of the newly synthesized derivatives were evaluated against a variety of pathogenic bacteria and fungi. Amikacin, ceftriaxone and penicillin belonging to aminoglycoside, cephalosporin and penicillin antibiotics, respectively, were used as positive antibacterial controls, as well as antifungal agents including terbinafine, fluconazole and nystatin. The antimicrobial effects were presented as IZD, MIC, MBC and MFC values in Tables 3 and 4.

According to the data reported in Table 3, the derivatives were ordered based on the spread of inhibitory properties and the MIC values as follows: $4 \mathrm{~b}>\mathbf{4 e}>\mathbf{4 d}>\mathbf{4 c}$ $>\mathbf{4 f}>\mathbf{4 a}$. The 3-phenyl ring in pyrazole derivative $\mathbf{4 b}$ was substituted by a methoxy group at para position, it was the only compound synthesized effective against Streptococcus pyogenes and Proteus vulgaris. The pyarazole 4a containing $p$-acetamidophenyl substituent was effective only against Gram-negative Salmonella typhi. The inhibitory effects of derivative $4 \mathbf{e}$ including 2,4-dichlorophenyl substituent were more significant than those of the derivative $\mathbf{4} \mathbf{f}$ with 2,6-dichlorophenyl substituent. Among pyrazoles $4 a-\mathbf{f}$, the antibacterial properties against Proteus mirabilis and 
Table 3. Antibacterial effects of synthesized pyrazoles and antibiotics.

\begin{tabular}{|c|c|c|c|c|c|c|c|c|c|c|}
\hline Products & Bacteria & $4 a$ & $4 b$ & $4 c$ & $4 d$ & $4 e$ & $4 f$ & $\mathrm{AMK}^{\mathrm{a}}$ & $\mathrm{CRO}^{\mathrm{b}}$ & PEN $^{c}$ \\
\hline \multirow{3}{*}{1768} & $\mathrm{IZD}^{\mathrm{d}}$ & - & 9.23 & 8.53 & & & - & 17.20 & - & - \\
\hline & $\mathrm{MIC}^{\mathrm{e}}$ & - & 512 & 512 & - & - & - & 2 & - & - \\
\hline & $M C^{f}$ & - & 1024 & 1024 & - & - & - & 8 & - & - \\
\hline \multirow{3}{*}{1447} & IZD & - & - & - & - & - & 15.46 & 20.17 & 25.88 & 22.61 \\
\hline & MIC & - & - & - & - & - & 64 & 1 & 0.5 & 0.25 \\
\hline & $\mathrm{MBC}$ & - & - & - & - & - & 128 & 4 & 1 & 0.5 \\
\hline \multirow{3}{*}{1709} & IZD & - & 10.11 & - & 11.50 & 10.10 & 11.51 & 10.10 & 32.64 & 14.03 \\
\hline & MIC & - & 256 & - & 256 & 512 & 256 & 0.5 & 2 & 4 \\
\hline & $\mathrm{MBC}$ & - & 512 & - & 512 & 1024 & 512 & 1 & 8 & 16 \\
\hline \multirow{3}{*}{1188} & IZD & - & 15.87 & - & - & - & - & 20.98 & - & - \\
\hline & MIC & - & 256 & - & - & - & - & 0.063 & - & - \\
\hline & $\mathrm{MBC}$ & - & 128 & - & - & - & - & 0.125 & - & - \\
\hline \multirow{3}{*}{1234} & IZD & - & - & - & 13.26 & - & 15.01 & 7.66 & 34.08 & 18.28 \\
\hline & MIC & - & - & - & 1024 & - & 512 & 0.5 & 2 & 8 \\
\hline & MBC & - & - & - & 2048 & - & 1024 & 4 & 4 & 16 \\
\hline \multirow{3}{*}{1776} & IZD & - & - & - & 10.74 & - & - & 14.65 & 33.91 & 20.73 \\
\hline & MIC & - & - & - & 128 & - & - & 0.25 & 0.063 & 8 \\
\hline & $\mathrm{MBC}$ & - & - & - & 256 & - & - & 4 & 1 & 32 \\
\hline \multirow{3}{*}{1609} & IZD & 12.21 & 13.89 & - & 11.67 & 14.78 & - & 19.31 & 30.43 & 10.95 \\
\hline & MIC & 64 & 64 & - & 64 & 32 & - & 0.063 & 0.063 & 4 \\
\hline & $\mathrm{MBC}$ & 128 & 128 & - & 128 & 64 & - & 0.25 & 0.125 & 16 \\
\hline \multirow{3}{*}{1435} & IZD & - & 17.76 & 12.14 & - & 13.82 & - & 20.71 & 18.54 & 23.58 \\
\hline & MIC & - & 16 & 64 & - & 64 & - & 0.25 & 0.5 & 0.5 \\
\hline & $\mathrm{MBC}$ & - & 32 & 128 & - & 256 & - & 4 & 2 & 1 \\
\hline \multirow{3}{*}{1310} & IZD & - & 12.10 & 13.67 & - & 14.10 & - & 19.07 & 16.21 & - \\
\hline & MIC & - & 128 & 16 & - & 16 & - & 0.063 & 0.5 & - \\
\hline & $\mathrm{MBC}$ & - & 256 & 32 & - & 32 & - & 0.063 & 1 & - \\
\hline \multirow{3}{*}{1240} & IZD & - & 12.16 & - & 11.55 & 11.34 & - & 17.44 & - & 12.20 \\
\hline & MIC & - & 256 & - & 256 & 256 & - & 1 & - & 8 \\
\hline & $\mathrm{MBC}$ & - & 512 & - & 512 & 512 & - & 1 & - & 16 \\
\hline \multirow{3}{*}{1079} & IZD & - & 11.78 & - & - & - & - & 22.42 & - & 12.82 \\
\hline & MIC & - & 16 & - & - & - & - & 4 & - & 8 \\
\hline & $\mathrm{MBC}$ & - & 64 & - & - & - & - & 4 & - & 32 \\
\hline \multirow{3}{*}{1633} & IZD & - & 19.45 & 11.92 & 11.08 & 15.20 & 9.52 & 19.47 & 21.51 & 17.29 \\
\hline & MIC & - & 32 & 32 & 256 & 128 & 128 & 1 & 2 & 8 \\
\hline & $\mathrm{MBC}$ & - & 64 & 128 & 512 & 256 & 256 & 2 & 2 & 16 \\
\hline
\end{tabular}

-: No noticeable antibacterial effects at selected highest concentration. ${ }^{a}$ Amikacin, ${ }^{\mathrm{b}}$ Ceftriaxone, ${ }^{\mathrm{c}}$ Penicillin, ${ }^{\mathrm{d}}$ Inhibition zone diameter in mm, ${ }^{\mathrm{e}}$ Minimum inhibitory concentration in $\mu \mathrm{g} / \mathrm{mL},{ }^{\mathrm{f}}$ Minimum bactericidal concentration in $\mu \mathrm{g} / \mathrm{mL}$.

Shigella dysenteriae were observed for the compounds $\mathbf{4 d}$ and $\mathbf{4 f}$, respectively. Amikacin in comparison with two other antibiotics could block the growth of all bacteria.

The in vitro antifungal activities of prepared pyrazoles were also evaluated and the results were promising. No inhibitory effect was observed with derivative $\mathbf{4 d}$ containing 2-hydroxy-3-methoxyphenyl substituent at the 3-position of the pyrazole ring. The dichloro compounds $4 \mathbf{e}$ and $4 \mathbf{f}$ had the same antifungal properties despite their different stereochemistry. Data gathered in Table 4 show that terbinafine has more remarkable effects than the others.

\section{Conclusions}

An efficient, one-pot two-step procedure was proposed and the synthesis of polysubstituted pyrazoles has been carried out. Some deep eutectic solvents including different molar ratios of potassium carbonate to glycerol were prepared and applied as reaction media and catalyst in this synthesis. The best results in terms of product yields and reaction times were achieved in molar ratios 1:4:14 of $\mathrm{K}_{2} \mathrm{CO}_{3} /$ glycerol/ $\mathrm{H}_{2} \mathrm{O}$. Efficiency of DES $\mathrm{K}_{2} \mathrm{CO}_{3} /$ glycerol in organic synthesis is currently under our investigation, and will be in focus of our future research. Furthermore, antimicrobial ac- 
Table 4. Antifungal effects of synthesized pyrazoles and drugs.

\begin{tabular}{|c|c|c|c|c|c|c|c|c|c|c|}
\hline Products & Fungi & $4 a$ & $4 b$ & $4 c$ & $4 d$ & $4 e$ & $4 f$ & TRB $^{\mathrm{a}}$ & FLC $^{\mathbf{b}}$ & NYT $^{\mathrm{c}}$ \\
\hline \multirow{3}{*}{5115} & $\mathrm{IZD}^{\mathrm{d}}$ & 11.56 & - & - & - & 15.25 & 15.29 & 23.94 & 15.23 & 20.45 \\
\hline & $\mathrm{MIC}^{\mathrm{e}}$ & 64 & - & - & - & 32 & 32 & 32 & 128 & 64 \\
\hline & $\mathrm{MBC}^{\mathrm{f}}$ & 128 & - & - & - & 64 & 64 & 64 & 256 & 128 \\
\hline \multirow{3}{*}{5027} & IZD & 21.87 & 14.77 & 14.71 & - & - & - & 36.24 & 14.81 & - \\
\hline & MIC & 64 & 32 & 32 & - & - & - & 32 & 256 & - \\
\hline & $\mathrm{MBC}$ & 128 & 64 & 64 & - & - & - & 64 & 512 & - \\
\hline \multirow{3}{*}{5009} & IZD & - & 23.57 & - & - & - & - & 29.18 & 21.13 & 20.52 \\
\hline & MIC & - & 512 & - & - & - & - & 32 & 32 & 32 \\
\hline & $\mathrm{MBC}$ & - & 1024 & - & - & - & - & 32 & 64 & 128 \\
\hline
\end{tabular}

-: No noticeable antibacterial effects at selected highest concentration. ${ }^{\mathrm{a}}$ Terbinafine, ${ }^{\mathrm{b}}$ Fluconazole, ${ }^{\mathrm{c}}$ Nystatin, ${ }^{\mathrm{d}}$ Inhibition zone diameter in mm, ${ }^{\mathrm{e}}$ Minimum inhibitory concentration in $\mu \mathrm{g} / \mathrm{mL},{ }^{\mathrm{f}}$ Minimum fungicidal concentration in $\mu \mathrm{g} / \mathrm{mL}$.

tivities of all synthesized derivatives were evaluated against a broad range of pathogenic bacteria and fungi. Based on the broad-spectrum inhibitory effects of the pyrazole $4 \mathbf{b}$, including 4-methoxy group on 3-aryl ring, it is suggested that benzaldehydes with small para electron donating substituents should be used to synthesize future active analogues.

\section{Acknowledgements}

This work was supported by the University of Zabol under Grant number UOZ-GR-9517-15.

\section{References}

1. F. F. Noe, L. Fowden, Nature 1959, 184, 69-70. DOI: $10.1038 / 184069 \mathrm{a} 0$

2. A. A. Wube, E. M. Wenzig, S. Gibbsons, K. Asres, R. Bauer, F. Bucar, Phytochemistry 2008, 69, 982-987.

DOI:10.1016/j.phytochem.2007.11.001

3. S. Domiati, A. El-Mallah, A. Ghoneim, A. Bekhit, H. A. El Razik, Inflammopharmacology 2016, 24, 163-172.

DOI:10.1007/s10787-016-0270-7

4. G. Cocconcelli, E. Diodato, A. Caricasole, G. Gaviraghi, E. Genesio, C. Ghiron, L. Magnoni, E. Pecchioli, P. V. Plazzi, G. C. Terstappen, Bioorg. Med. Chem. 2008, 16, 2043-2052. DOI:10.1016/j.bmc.2007.10.090

5. M. J. Ahsan, H. Khalilullah, J. P. Stables, J. Govindasamy, J. Enzyme Inhib. Med. Chem. 2013, 28, 644-650.

DOI:10.3109/14756366.2012.663364

6. M. Bonesi, M. R. Loizzo, G. A. Statti, S. Michel, F. Tillequin, F. Menichini, Bioorg. Med. Chem. Lett. 2010, 20, 1990-1993. DOI:10.1016/j.bmcl.2010.01.113

7. K. M. Kasiotis, E. N. Tzanetou, S. A. Haroutounian, Front. Chem. 2014, 2, 78 (7 pp). DOI:10.3389/fchem.2014.00078

8. Y.-F. Li, Z.-Q. Liu, Free Radic. Biol. Med. 2011, 52, 103-108. DOI:10.1016/j.freeradbiomed.2011.09.032

9. D. Swarnkar, R. Ameta, R. Vyas, European J. Biomed. Pharm. Sci. 2016, 3, 427-431.
10. R. E. El-Mekawy, J. Heterocycl. Chem. 2017, 54, 2367-2374. DOI: $10.1002 /$ jhet. 2828

11. S. Y. Hassan, Molecules 2013, 18, 2683-2711. DOI:10.3390/molecules18032683

12. R. Surendra Kumar, I. A. Arif, A. Ahamed, A. Idhayadhull, Saudi J. Biol. Sci. 2016, 23, 614-620.

DOI:10.1016/j.sjbs.2015.07.005

13. K. M. El-Mahdy, A. M. El-Kazak, M. Abdel-Megid, M. Seada, O. Farouk, Acta Chim. Slov. 2016, 63, 18-25.

DOI:10.17344/acsi.2015.1555

14. F. Gosselin, P. D. O'Shea, R. A. Webster, R. A. Reamer, R. D. Tillyer, E. J. J. Grabowski, Synlett 2006, 2006, 3267-3270.

DOI:10.1055/s-2006-956487

15. Y. Kong, M. Tang, Y. Wang, Org. Lett. 2014, 16, 576-579. DOI: $10.1021 / \mathrm{ol} 403447 \mathrm{~g}$

16. D. C. Schmitt, A. P. Taylor, A. C. Flick, R. E. Kyne Jr., Org. Lett. 2015, 17, 1405-1408. DOI:10.1021/acs.orglett.5b00266

17. Q. Zhang, L.-G. Meng, K. Wang, L. Wang, Org. Lett. 2015, 17, 872-875. DOI:10.1021/ol503735c

18. R. M. Mohareb, N. Y. M. Abdo, F. O. Al-farouk, Acta Chim. Slov. 2017, 64, 117-128. DOI:10.17344/acsi.2016.2920

19. J.-A. Jiang, C.-Y. Du, C.-H. Gu, Y.-F. Ji, Synlett 2012, 23, 29652968. DOI:10.1055/s-0032-1317668

20. A. Kamal, K. N. V. Sastry, D. Chandrasekhar, G. S. Mani, P. R. Adiyala, J. B. Nanubolu, K. J. Singarapu, R. A. Maurya, J. Org. Chem. 2015, 80, 4325-4335. DOI:10.1021/jo502946g

21. N. Panda, A. K. Jena, J. Org. Chem. 2012, 77, 9401-9406. DOI:10.1021/jo301770k

22. G. C. Senadi, W.-P. Hu, T.-Y. Lu, A. M. Garkhedkar, J. K. Vandavasi, J.-J. Wang, Org. Lett. 2015, 17, 1521-1524.

DOI:10.1021/acs.orglett.5b00398

23. S. Specklin, E. Decuypere, L. Plougastel, S. Aliani, F. Taran, J. Org. Chem. 2014, 79, 7772-7777. DOI:10.1021/jo501420r

24. L.-L. Wu, Y.-C. Ge, T. He, L. Zhang, X.-L. Fu, H.-Y. Fu, H. Chen, R.-X. Li, Synthesis 2012, 44, 1577-1583.

DOI:10.1055/s-0031-1290772

25. A. Hasaninejad, S. Firoozi, Mol. Divers. 2013, 17, 459-469. DOI:10.1007/s11030-013-9445-y

26. S. Kumari, A. Shekhar, D. D. Pathak, New J. Chem. 2016, 40, 5053-5060. DOI:10.1039/C5NJ03380B 
27. M. O. M'hamed, O. K. Alduaij, Asian J. Chem. 2016, 28, $543-$ 547. DOI:10.14233/ajchem.2016.19397

28. F. Nemati, S. H. Nikkhah, A. Elhampour, Chin. Chem. Lett. 2015, 26, 1397-1399. DOI:10.1016/j.cclet.2015.07.009

29. S. Yadav, P. Rai, M. Srivastava, J. Singh, K. P. Tiwari, J. Singh, Tetrahedron Lett. 2015, 56, 5831-5835.

DOI:10.1016/j.tetlet.2015.07.039

30. M. Capua, S. Perrone, F. M. Perna, P. Vitale, L. Troisi, A. Salomone, V. Capriati, Molecules 2016, 21, 924 (11 pp).

DOI:10.3390/molecules21070924

31. S. B. Katariya, Asian J. Pharm. Sci. Tech. 2015, 5, 199-201.
32. A. Moshtaghi Zonouz, D. Moghani, Synth. Commun. 2016, 46, 220-225. DOI:10.1080/00397911.2015.1129668

33. J. Naser, F. Mjalli, B. Jibril, S. Al-Hatmi, Z. Gano, Int. J. Chem. Eng. Appl. 2013, 4, 114-118.

DOI:10.7763/IJCEA.2013.V4.275

34. H. Beyzaei, M. Moghaddam-Manesh, R. Aryan, B. Ghasemi, A. Samzadeh-Kermani, Chem. Pap. 2017, 71, 1685-1691. DOI:10.1007/s11696-017-0163-2

35. S. Arikan, Med. Mycol. 2007, 45, 569-587. DOI:10.1080/13693780701436794

\section{Povzetek}

Različne biološke lastnosti naravnih in sintetičnih pirazolskih derivatov, kot so npr. delovanja proti vnetjem in mikrobom, nevrozaščitni učinki, antiepileptični ter antidepresivni učinki in aktivnosti proti rakom, so nas spodbudili, da smo predlagali novo, hitro, zeleno in ekološko sprejemljivo pot za pripravo nekaterih novih 5-amino-3-(aril substituiranih)-1-(2,4-dinitrofenil)-1H-pirazol-4-karbonitrilov. Učinkovito smo jih pripravili z enolončno dvostopenjsko reakcijo med malononitrilom, 2,4-dinitrofenilhidrazinom in različnimi benzaldehidi v globoko evtektičnem topilu (DES) glicerol/kalijev karbonat. Uporaba tovrstnega topilnega sistema je opazno povečala izkoristke produktov in skrajšala reakcijske čase. Raziskali smo antibakterijsko delovanje novopripravljenih pirazolov in rezultate primerjali z učinki več običajnih antibiotikov na izbrane Gram-pozitivne in Gram-negativne patogene bakterije. Raziskali smo tudi inhibitorno aktivnost proti trem glivam in jo primerjali z nekaterimi običajnimi učinkovinami proti glivam. Zmerno do dobro antimikrobno delovanje, predvsem pa delovanje proti glivam, smo opazili v nekaterih primerih naših heterocikličnih spojin, kot je bilo razvidno iz izmerjenih IZD, MIC, MBC in MFC vrednosti. 